\title{
Distributed Service and Network Management Using Intelligent Filters
}

\author{
C. Cicalese, (703)883-1914, (703)883-5862 (fax), cindy@mitre.org \\ J. DeCarlo, (703) 883-7116, (703) 883-5862 (fax), jdecarlo@mitre.org \\ M. Kahn, (703)883-7356, (703)883-5862 (fax), mkahn@mitre.org \\ E.D.Zeisler, (703) 883-5768, (703) 883-5862 (fax), ezeisler@mitre.org \\ The MITRE Corporation \\ 1820 Dolley Madison Blvd., Mail Stop W658 \\ McLean, VA 22102-3481 USA
}

H.Folts, (301)614-5229, (301)614-0267 (fax),folts@eos.nasa.gov NASA Goddard Space Flight Center

Building 32, Room N230B

Greenbelt, MD 20771 USA

\begin{abstract}
This work addresses two capabilities important to the management of distributed services. First, in order to be able to determine the root cause of a service-affecting fault, network management information must be correlated with service management information. Second, since distributed services often span many management domains, it must be possible to communicate trouble information securely from one management domain to another.

A prototype was developed to demonstrate how these ideas could be applied to managing NASA's Earth Observing System Data and Information System (EOSDIS). The prototype integrated commercial off-the-shelf products such as Hewlett-Packard's OpenView and Remedy's Action Request System with the Open Group's Distributed Computing Environment (DCE). The event correlation component was constructed using the C Language Integrated Production System (CLIPS). The service manager collected management information from the simulated service and network elements by a combination of polling and sending. Upon determination of the root cause of a fault, a trouble ticket could be automatically generated and, if necessary, could be forwarded securely to the appropriate management domain using an authenticated DCE remote procedure call.
\end{abstract}

